05,13

\title{
Структура и магнетизм сверхрешеток Co/Dy
}

\author{
(C) М.В. Макарова ${ }^{1,2}$, Е.А. Кравцов ${ }^{1,2}$, В.В. Проглядо ${ }^{1}$, Yu. Khaydukov ${ }^{3,4}$, B.В. Устинов ${ }^{1,2}$ \\ ${ }^{1}$ Институт фозиики металлов УрО РАН, \\ Екатеринбург, Россия \\ ${ }^{2}$ Уральский федеральный университет, \\ Екатеринбург, Россия \\ ${ }^{3}$ Max Planck Institute for Solid State Physics, \\ Stuttgart, 70569 Germany \\ ${ }^{4}$ Max Planck Society Outstation at Heinz Maier-Leibnitz Zentrum, \\ Garching, Germany \\ E-mail: kravtsov@imp.uran.ru
}

Поступила в Редакцию 26 марта 2020 г.

В окончательной редакции 26 марта 2020 г.

Принята к публикации 2 апреля 2020 г.

Исследовано влияние структурных свойств на формирование перпендикулярной магнитной анизотропии в сверхрешетках $\mathrm{Co} / \mathrm{Dy}$. Установлено, что сверхрешетки представляют собой композиционно- модулированный сплав, в котором, с одной стороны, выдерживается строгая периодичность в толщинах и композиции слоев, а с другой стороны, интердиффузия Со и Dу на межслойных границах приводит к периодическому изменению с глубиной концентрации Со и Dу. Показано, что перпендикулярная магнитная анизотропия в $\mathrm{Co} / \mathrm{Dy}$ обусловлена перпендикулярной ориентацией намагниченности в слоях сплава СоDy, в то время как намагниченность слоев Со ориентирована в плоскости образца.

Ключевые слова: металлические магнитные сверхрешетки, межслойное магнитное упорядочение, перпендикулярная магнитная анизотропия, рефлектометрия поляризованных нейтронов.

DOI: $10.21883 /$ FTT.2020.09.49776.15H

\section{1. Введение}

Многослойные наноструктуры, образованные чередующимися слоями магнитных редкоземельных и переходных металлов (РЗМ/ПМ) - популярные объекты исследований в современной спинтронике, для которых характерно проявление ряда уникальных магнитных свойств. Во-первых, поскольку магнитные моменты тяжелых РЗМ и ПМ упорядочиваются антипараллельно, а температуры Кюри и магнитные моменты этих металлов различны, то, варьируя толщины слоев и температуру, можно в широких пределах изменять магнитное упорядочение в таких системах, в том числе добиваться компенсации магнитных моментов РЗМ и ПМ подрешеток. Во-вторых, в РЗМ/ПМ наноструктурах со сравнительно тонкими слоями возможно наличие перпендикулярной магнитной анизотропии (ПМА). Магнитные наноструктуры, в которых можно обеспечить магнитную компенсацию и наблюдается ПМА, сегодня широко востребованы в различных устройствах магнитной спинтроники, например, в магнитоптических и термических системах записи и хранения информации со сверхбыстрым переключением намагниченности, наноосцилляторах и др.

Первыми РЗМ/ПМ сверхрешетками, в которых ПМА наблюдалась для сравнительно толстых слоев (до $18 \AA$ ), были системы Co/Dy [1-3]. Недавно нами было показано, ПМА в сверхрешетках Co/Dy может наблюдаться и при более толстых слоях Со и Dy (до $30 \AA$ ) в зависимости от микроструктуры образцов [4]. В настоящей работе мы сообщаем об исследовании влияния кристаллической структуры на магнитную анизотропию и магнитное упорядочение сверхрешеток Co/Dy с различными толщинами слоев Со.

\section{2. Эксперимент}

Были исследованы две сверхрешетки $[\mathrm{Co}(12 \AA) / \mathrm{Dy}(12 \AA)]_{40}$ и $[\mathrm{Co}(30 \AA) / \mathrm{Dy}(12 \AA)]_{40}$, выращенные методом высоковакуумного магнетронного распыления на монокристаллических подложках (001)Si c буферным слоем $\mathrm{Nb}$ толщиной $100 \AA$ и защитным слоем Al толщиной $100 \AA$. Синтез образцов проводился при комнатной температуре, рост многослойной структуры на поверхности буферного слоя $\mathrm{Nb}$ начинался со слоя Dy и заканчивался слоем Co. Рентгеноструктурные исследования были выполнены в геометрии параллельного пучка на лабораторном дифрактометре PANalytical Empyrean Series 2 с использованием $\mathrm{Fe}-K_{\alpha}$ и $\mathrm{Co}-K_{\alpha}$ излучения. Магнитометрические измерения выполнены на вибрационном магнетометре Lake Shore 7407 в магнитных полях до $15 \mathrm{kOe}$ и при температурах от комнатной до $70 \mathrm{~K}$. Измерения рефлектометрии поляризованных нейтронов были выполнены в стандартной $\theta-2 \theta$ геометрии на рефлектометре NREX на исследовательском реакторе FRM II (Гархинг, Германия). Измерения с полным поляризационным анализом 
проведены на длине волны $4.26 \pm 0.06 \AA$, эффективность поляризатора была $99.99 \%$, анализатора - $98 \%$.

\section{3. Результаты и обсуждение}

Свойства слоистой структуры образцов и структуры межслойных границ были определены с помощью рентгеновской рефлектометрии высокого разрешения. На рис. 1 представлены результаты рефлектометрических исследований сверхрешеток и результаты их обработки (фитирования), для удобства представления рефлектометрические кривые для разных образцов умножены на различные постоянные коэффициенты. Из обработки рефлектометрических кривых установлено, что, с одной стороны, в сверхрешетках сохраняется строгая периодичность слоистой структуры, а с другой стороны, наблюдается значительное перемешивание Dy и Со на межслойных границах, толщина переходного слоя DуСо может достигать $8 \AA$ А. Таким образом, можно утверждать что сверхрешетки представляют собой композиционно-модулированный сплав с периодически изменяющейся с глубиной концентрацией Со и Dy. В образце $\mathrm{Co}(12 \AA) / \mathrm{Dy}(12 \AA)$ нами не было обнаружено сплошных слоев Dу и Со, в образце Co(30 ̊)/Dy $(12 \AA)$ присутствует как сплав DуCo, так и сплошной слой Сo толщиной $19 \AA$.

Кристаллическая структура образцов была определена с помощью рентгеновской дифракции в геометрии скользящего падения (угол падения $1^{\circ}$ ) с использованием Co$K_{\alpha}$-излучения. На рис. 2 представлены дифрактограммы, измеренные для обоих образцов. Из дифрактограмм видно, что в скане от образца $\mathrm{Co}(12 \AA) / \mathrm{Dy}(12 \AA)$ присутствует исключительно сигнал от сплава $\mathrm{DyCo}_{3}(11-20)$, что согласуется с данными рентгеновской рефлектометрии. На дифрактограмме от $\mathrm{Co}(30 \AA) / \mathrm{Dy}(12 \AA)$ присут-

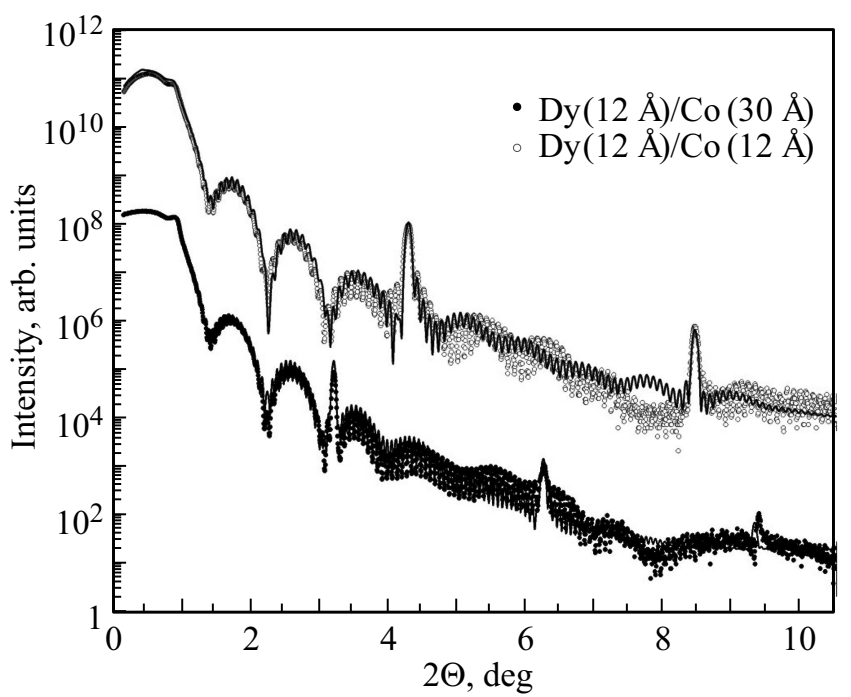

Рис. 1. Кривые рентгеновской рефлектометрии (символы) и результаты их обработки (сплошные линии) для сверхрешеток $\mathrm{Co}(12 \AA) / \mathrm{Dy}(12 \AA)$ и $\mathrm{Co}(30 \AA) / \operatorname{Dy}(12 \AA)$.

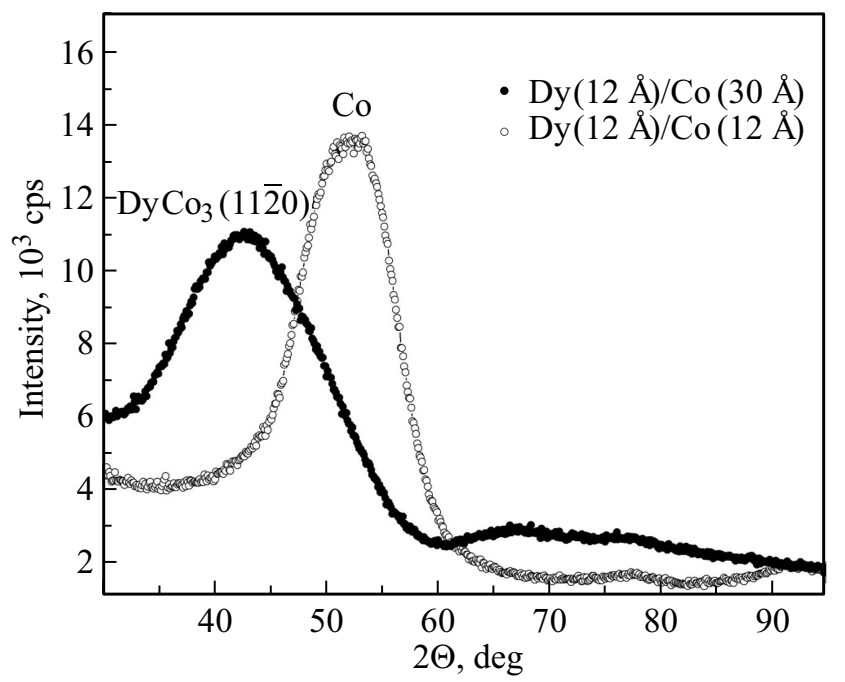

Рис. 2. Рентгеновские дифрактограммы для сверхрешеток $\mathrm{Co}(12 \AA) / \mathrm{Dy}(12 \AA)$ и $\mathrm{Co}(30 \AA) / \mathrm{Dy}(12 \AA)$, измеренные в геометрии скользящего падения.

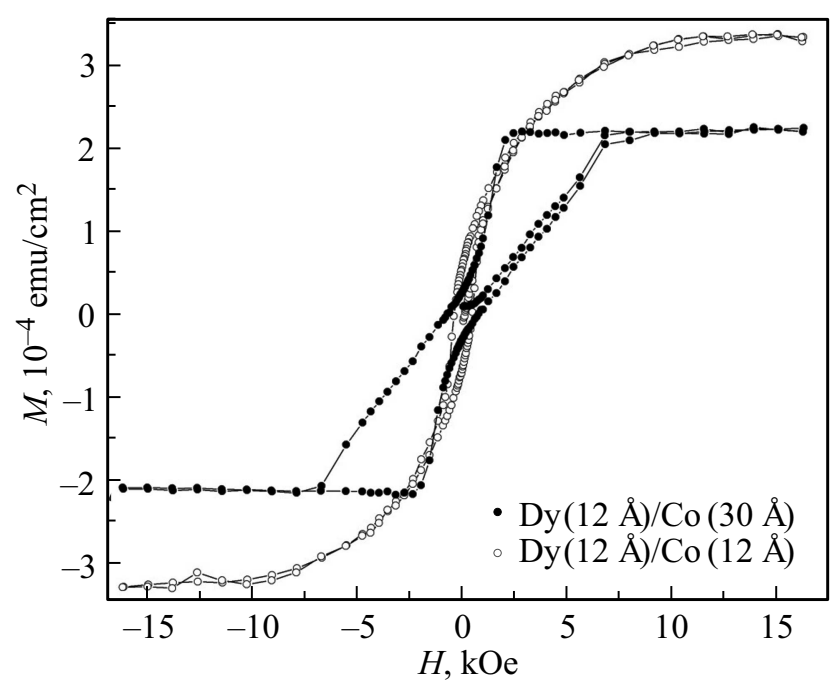

Рис. 3. Петли гистерезиса для сверхрешеток $\mathrm{Co}(12 \AA) / \mathrm{Dy}(12 \AA)$ и $\mathrm{Co}(30 \AA) / \mathrm{Dy}(12 \AA)$, измеренные при температуре $T=80 \mathrm{~K}$ при ориентации магнитного поля вдоль нормали к поверхности.

ствует сигнал от Со. К сожалению, пик на дифрактограмме достаточно широкий, что не позволяет установить кристаллографическую ориентацию Со: сигнал может быть как от ГПУ $\mathrm{Co}(0002)$, так и от ГЦК $\mathrm{Co}(111)$. Тем не менее, данные рентгеновской дифрактометрии однозначно подтверждают наличие чистого кристаллического Со в образце $\mathrm{Co}(30 \AA) / \mathrm{Dy}(12 \AA)$ и полное его отсутствие в $\mathrm{Co}(12 \AA) / \mathrm{Dy}(12 \AA)$.

Комбинированные магнитные и нейтронографические измерения показали, что намагниченность сверхрешетки $\mathrm{Co}(12 \AA) / \mathrm{Dy}(12 \AA)$ при всех температурах ориентирована перпендикулярно поверхности образца, в то время как в образце $\mathrm{Co}(30 \AA) / \mathrm{Dy}(12 \AA)$ она направлена под 


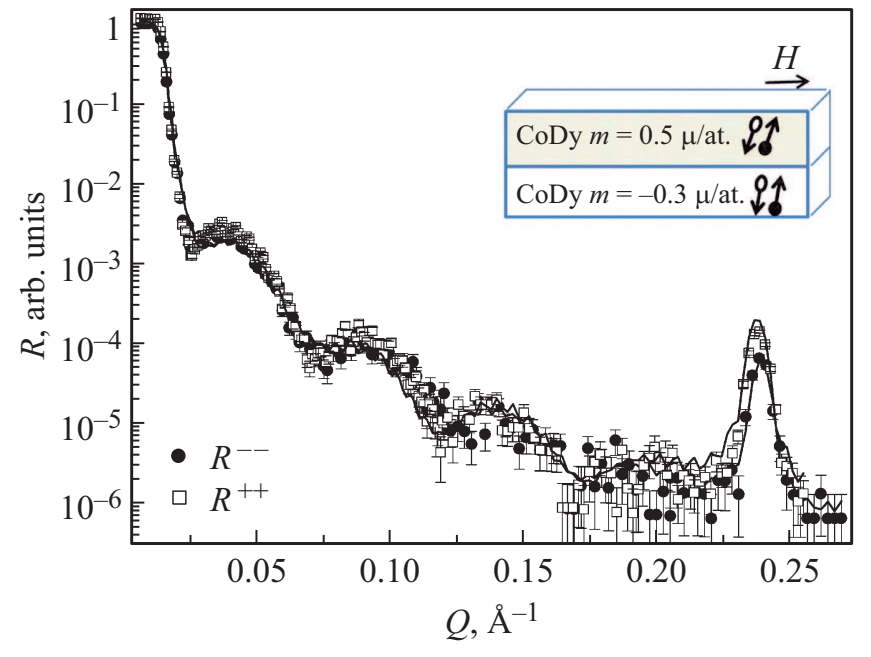

Рис. 4. Кривые рефлектометрии поляризованных нейтронов (символы) и результаты их обработки (сплошные линии) для сверхрешетки $\mathrm{Co}(12 \AA) / \mathrm{Dy}(12 \AA)$, измеренные в магнитном поле $1.5 \mathrm{kOe}$ при температуре $T=80 \mathrm{~K}$.

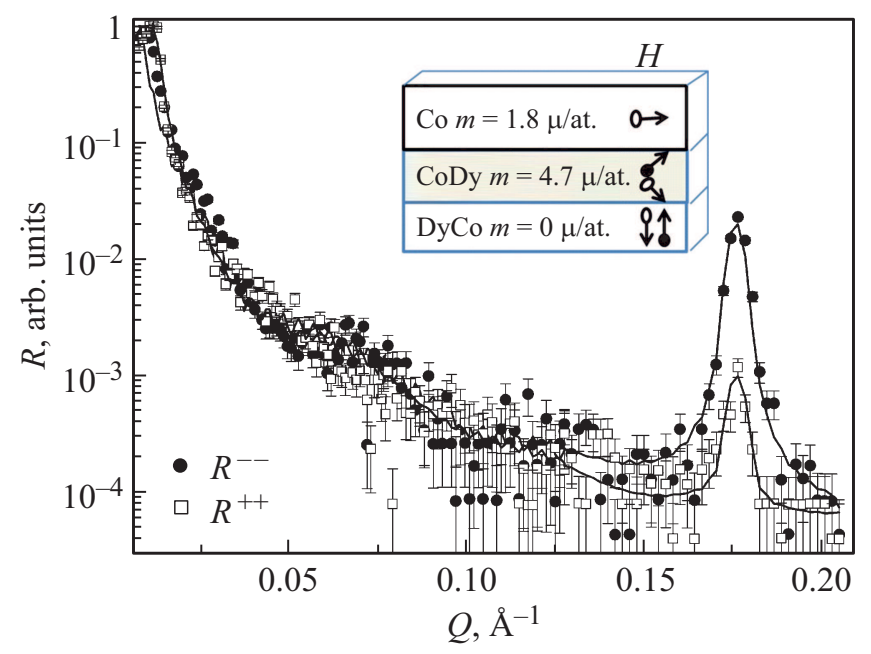

Рис. 5. Кривые рефлектометрии поляризованных нейтронов (символы) и результаты их обработки (сплошные линии) для сверхрешетки $\mathrm{Co}(30 \AA) / \mathrm{Dy}(12 \AA)$, измеренные в магнитном поле $1.5 \mathrm{kOe}$ при температуре $T=80 \mathrm{~K}$.

некоторым углом к нормали. На рис. 3 представлены петли гистерезиса для обоих образцов, измеренные при ориентации магнитного поля вдоль нормали к поверхности при температуре $T=80 \mathrm{~K}$ (несколько выше температуры объемного Dy). Петля гистерезиса сверхрешетки $\mathrm{Co}(12 \AA) / \mathrm{Dy}(12 \AA)$ свидетельствует об ориентации магнитных моментов преимущественно перпендикулярно поверхности, в то время как в сверхрешетке $\mathrm{Co}(30 \AA) / \mathrm{Dy}(12 \AA)$ вдоль нормали к поверхности ориентирована ось трудного намагничивания. Магнитные свойства образца $\mathrm{Co}(30 \AA) / \mathrm{Dy}(12 \AA)$ значительно отличаются от свойств системы $\mathrm{Co}(30 \AA) / \mathrm{Dy}(20 \AA)$, в которой при температуре $T=90 \mathrm{~K}$ магнитные моменты были ориентированы вдоль нормали к поверхности и лишь при $T=150 \mathrm{~K}$ происходит их переориентация [4]. Таким образом, уменьшение толщины слоев Dy с $20 \AA$ до $12 \AA ̊$ приводит к ослаблению перпендикулярной магнитной анизотропии и уменьшению температуры ориентационного перехода от перпендикулярной к параллельной ориентации относительно нормали к поверхности.

На рис. 4 и 5 представлены результаты измерений рефлектометрии поляризованных нейтронов для обоих образцов, выполненные при температуре $80 \mathrm{~K}$ в магнитном поле $1.5 \mathrm{kOe}$. Анализ рефлектограмм показал, что в образце $\mathrm{Co}(12 \AA) / \mathrm{Dy}(12 \AA)$ сплошного слоя Со не образуется, а магнитные моменты в сплаве CoDy ориентированы перпендикулярно поверхности. В образце $\mathrm{Co}(30 \AA) / \mathrm{Dy}(12 \AA)$ частично образуется сплошной слой Co, магнитные моменты в котором лежат в плоскости образца, в то время как намагниченность CoDy слоя ориентирована вдоль нормали к поверхности. Таким образом, можно утверждать, что перпендикулярная магнитная анизотропия в сверхрешетках Co/Dy формируется в слоях сплава CoDy, в то время как магнитные моменты в слоях Со лежат в плоскости образца.

\section{4. Заключение}

Нами установлено, что сверхрешетки Co/Dy представляют собой композиционно-модулированный сплав, в котором, с одной стороны, выдерживается строгая периодичность в толщинах и композиции слоев, а с другой стороны, интердиффузия Со и Dу на межслойных границах приводит к периодическому изменению с глубиной концентрации Со и Dу. Показано, что перпендикулярная магнитная анизотропия в Co/Dy обусловлена перпендикулярной ориентацией намагниченности в слоях CoDy, в то время как намагниченность слоев Со ориентирована в плоскости образца.

\section{Благодарности}

Синтез образцов, магнитометрические и рентгеноструктурные измерения выполнены в Центре коллективного пользования ИФМ УрО РАН. Эксперименты по рефлектометрии поляризованных нейтронов выполнены на инструменте NREX, действующем на стационарном реакторе FRM-II в Heinz Maier-Leibnitz Center (Гархинг, Германия), который используется Институтом Макса Планка по физике твердого тела (Штутгарт, Германия).

\section{Финансирование работы}

Исследование выполнено в рамках государственного задания Министерства науки и высшего образования Российской Федерации (тема „Спин“ № АААА-А18118020290104-2) при частичной поддержке РФФИ (проекты № 20-42-660024 и 19-02-00057).

\section{Конфликт интересов}

Авторы заявляют, что у них нет конфликта интересов. 


\section{Список литературы}

[1] Z.S. Shan, D.J. Sellmyer, S.S. Jaswal, Y.J. Wang, J.X. Shen. Phys. Rev. Lett. 63, 449 (1989).

[2] Z.S. Shan, D.J. Sellmyer. Phys. Rev. B 42, 10433 (1990).

[3] Z.S. Shan, D.J. Sellmyer, S.S. Jaswal, Y.J. Wang, J.X. Shen. Phys. Rev. B 42, 10446 (1990).

[4] I.A. Subbotin, E.M. Pashaev, A.L. Vasiliev, Yu M. Chesnokov, G.V. Prutskov, E.A. Kravtsov, M.V. Makarova, V.V. Proglyado, V.V. Ustinov. Physica B 573, 28 (2019).

Редактор Т.Н. Василевская 